\title{
Comportamiento de ecoladrillos con inclusión de biomasas residuales
}

\begin{abstract}
Extracto:
El ladrillo cerámico como material de construcción industrial contribuye negativamente al deterioro del medioambiente, tanto por el agotamiento de los recursos naturales como por la elevada cantidad de energía necesaria para su fabricación, que se traduce en emisiones de $\mathrm{CO}_{2}$. La incorporación de productos residuales o subproductos procedentes de diversos procesos industriales ha dado lugar a los denominados «ecoladrillos». En este trabajo de investigación se estudia el comportamiento de ladrillos cerámicos con la incorporación de diferentes biomasas residuales con la función de generar una red porosa que modifique sus propiedades para adaptarlos a los requerimientos técnicos actuales. Para ello se han fabricado 19 tipos de ladrillos con la incorporación de 6 subproductos biomásicos: cascarilla de arroz (C), cáscara de almendra (CA), hueso de aceituna (HA), hoja de olivo $(\mathrm{HO}$, ) leña de olivo (LO) y poda de olivo (PO). Las biomasas se han incorporado a una arcilla convencional en tres proporciones en volumen $(7,5 \%, 15 \%$ y $25 \%$ ), con tres tamaños de distribución de partícula (0/1 mm, $1 / 2 \mathrm{~mm}$ y 0/2 mm). Los resultados obtenidos muestran que el tamaño de partícula no ha condicionado el comportamiento físico y mecánico de los ecoladrillos, no así la proporción de biomasa, aunque en ningún caso se ha comprometido la normal funcionalidad de los mismos. Por el contrario, se ha observado una mejora considerable en la conductividad térmica de los ecoladrillos fabricados, fundamentalmente, con los menores porcentajes de reemplazo.
\end{abstract}

Palabras clave: ladrillos cerámicos, biomasas residuales, propiedades físicas, propiedades mecánicas, economía circular.

\footnotetext{
${ }^{1}$ M. ${ }^{a}$ Martín-Morales, miembro del Departamento de Construcciones Arquitectónicas de la Universidad de Granada.

${ }^{2}$ D. Eliche-Quesada, miembro del Departamento de Ingeniería Química, Ambiental y de los Materiales de la Universidad de Jaén.

${ }^{3}$ M. López-Alonso, miembro del Departamento de Ingeniería de la Construcción y Proyectos de Ingeniería de la Universidad de Granada.

4 J. Martín-Pascual, miembro del Departamento de Ingeniería Civil de la Universidad de Granada.

${ }^{5}$ L. Pérez-Villarejo, miembro del Departamento de Ingeniería Química, Ambiental y de los Materiales de la Universidad de Jaén.

${ }^{6}$ D. P. Ruiz-Padillo, miembro del Departamento de Física Aplicada de la Universidad de Granada.

${ }^{7}$ M. Zamorano, miembro del Departamento de Ingeniería Civil de la Universidad de Granada.
}

Este artículo se enmarca dentro de la Convocatoria de Ayudas a Proyectos de 1+D+i 2016 de la Fundación Hergar (categoría: Investigación aplicada y tecnológica en ingenierías). Los autores quieren agradecer a la empresa Ladrillos Suspiro del Moro, SL la aportación de las materias primas y las dosificaciones necesarias para el desarrollo del estudio experimental. 


\section{Behavior of ecobricks with the inclusion of residual biomasses}

\section{Abstract:}

Industrially manufactured ceramic bricks have a negative effect on the environment due to both the depletion of natural resources and the $\mathrm{CO}_{2}$ emissions resulting from the considerable amount of energy used in their production. Recently, diverse waste or subproducts from different industrial processes have been used to improve the performance of ceramic materials, giving rise to the creation of the «ecobrick». In this study, we present the results obtained from experiments using various biomass waste products to create a porous network capable of modifying the properties of traditional bricks to adapt them to modern technical requirements. 19 type of brick were manufactured with the addition of 6 biomass subproducts: rice husks (C), almond husks $(\mathrm{CA})$, olive stones $(\mathrm{HA})$, olive leaves $(\mathrm{HO})$, olive wood (LO) and pruning waste from olive trees (PO). These materials were added to a conventional clay mix in three different proportions $(7.5 \%$, $15 \%$ and $25 \%)$ and three different particle size distributions $(0 / 1 \mathrm{~mm}, 1 / 2 \mathrm{~mm}$ and $0 / 2 \mathrm{~mm})$. The results show that particle size does not affect the performance (physical and mechanical) of the ecobricks. However, performance was negatively affected in proportion to the amount of biomass waste added to the mix, without this undermining the normal functionality of the bricks. In fact, a considerable improvement was observed in the thermal conductivity of the ecobricks. This was true particularly, and surprisingly, of the mix containing the lowest proportion of additional material.

Keywords: clay bricks, residual biomasses, physical properties, mechanical properties, circular economy.

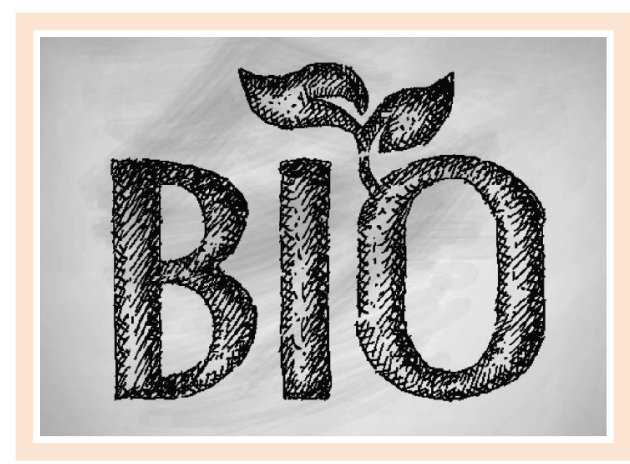

\section{INTRODUCCIÓN}

El sector de la construcción tiene un papel muy importante en el desarrollo social y económico de un país, pero también es una de las industrias que generan mayores repercusiones medioambientales negativas tanto por el agotamiento de los recursos naturales y el consumo de energía como por la gran cantidad de residuos y emisiones al ambiente. La industrialización del sector ha supuesto un enorme revulsivo en cuanto a la fabricación en serie de gran cantidad de productos acordes con las normativas técnicas vigentes pero con unas cargas medioambientales cada vez mayores.

En este sentido, uno de los productos de construcción más industrializados son los ladrillos cerámicos de arcilla cocida. Los ladrillos para la construcción, que inicialmente se fabricaban con arcilla prensada a mano (Lajo, 1990), mejoraron sustancialmente sus propiedades físicas y mecánicas tras un proceso de cocción. Las primeras referencias del uso de los ladrillos cocidos datan del 2500 a. C. (Chabat, 1881); así en la Antigua Grecia, donde se acuñó el término keramokos (quemado o cocido), tuvieron un gran uso.

\section{El sector de la construcción tiene un papel muy importante en el desarrollo social y económico de un país, pero también es una de las industrias que generan mayores repercusiones medioambientales negativas}


La revolución industrial afectó de manera muy considerable a la industrialización de la producción del ladrillo (Brongniart, 1844), consiguiéndose productos muy económicos con materias primas abundantes y baratas, como son las arcillas y arenas, mediante un proceso muy simple de fabricación por conformación mecánica en piezas de múltiples formas y tamaños, que alcanzan sus propiedades finales tras el secado y la cocción. Por este motivo el ladrillo cerámico experimentó un gran auge, convirtiéndose en uno de los elementos estructurales claves para el desarrollo de la mayoría de las infraestructuras edificatorias e industriales. Sin embargo, en la década de los ochenta, cuando el hormigón empieza a ser el producto estructural estrella, el ladrillo quedó relegado a labores de cerramiento y separación entre espacios, en algunos casos con carácter meramente decorativo.

Con la entrada en vigor de normativas relativas a mantener las condiciones mínimas de habitabilidad (NBE-FL 90, 1990; CTE, 2006), así como con los requerimientos actuales cada vez más exigentes en cuanto al ahorro energético, tipo passivhaus o consumo de energía casi nulo, los requerimientos de los materiales de construcción, en general, y de los ladrillos cerámicos, en particular, se han ido adaptando a las exigencias impuestas. El paulatino perfeccionamiento producido en estos materiales tradicionales ha estado condicionado tanto a la composición de las materias primas y al método de producción como al proceso de cocción y a la temperatura alcanzada.

En este sentido se han llevado a cabo diferentes investigaciones conducentes a incorporar determinados productos residuales o subproductos procedentes de diversos procesos industriales, de naturaleza inorgánica y orgánica, destinados a mejorar el comportamiento tecnológico de los materiales cerámicos, dando lugar a la creación del denominado «ecoladrillo». Se trata de un ladrillo cerámico que, además de la tradicional mezcla arcillosa, incorpora diferentes residuos al objeto de:

- Mejorar fundamentalmente su red porosa (Banhidi y Gömze, 2008).

- Provocar una reducción del gasto energético y, en consecuencia, un menor daño asociado a las emisiones generadas durante su fabricación (Demir, Serhat Baspinar y Orhan, 2005; Kazmi, Abbas, Saleem, Munir y Khitab, 2016).

- Reducir los costos de materias primas, incluyendo arcilla y agua (Devant, Cusidó y Soriano, 2010).
- Mejorar medioambientalmente su uso durante su etapa de servicio debido a sus mejores cualidades como aislante térmico.

- Finalmente, reducir la eliminación de residuos en vertederos, gracias a la valorización de los residuos utilizados (Arsenovic, Radojevic y Stankovic, 2012).

En algunos casos se ha constatado, además, que añadir determinados subproductos industriales a los ladrillos cerámicos aporta como ventaja adicional la inmovilización de metales pesados (Devant et al., 2010; Pérez-Villarejo et al., 2015).

Algunos ejemplos de trabajos experimentales realizados ponen de manifiesto el objetivo y los resultados obtenidos con la incorporación de diferentes residuos en las pastas cerámicas. En unos casos los investigadores han introducido residuos industriales inorgánicos al objeto de sustituir la arena como material fundente en la mezcla arcillosa. Con la incorporación de residuos inorgánicos, tales como arenas de fundición, en un porcentaje del 10 al $35 \%$ en peso (Alonso-Santurde et al., 2011), se han encontrado mejoras en la plasticidad de las pastas cerámicas, consiguiendo básicamente una disminución de la porosidad y, por ende, la absorción de agua. Similares resultados se consiguen con la inclusión de otros residuos inorgánicos en las mezclas arcillosas. Los lodos de plantas de tratamiento de efluentes de petróleo en hasta el $35 \%$ en peso (Sengupta, Saikia y Borthakur, 2002) reducen la necesidad de agua de amasado y actúan como combustible suplementario en el proceso de cocción de los ladrillos. Pérez-Villarejo y sus colaboradores (2015) emplearon un $5 \%$ en peso de lodos galvánicos obtenidos del tratamiento físico-
[...] se han llevado a cabo diferentes investigaciones conducentes a incorporar determinados productos residuales o subproductos procedentes de diversos procesos industriales [...] destinados a mejorar el comportamiento tecnológico de los materiales cerámicos, dando lugar a la creación del «ecoladrillo» 
químico de aguas residuales en sustitución de la arcilla convencional, obteniendo mejoras considerables en la porosidad y resistencia mecánica con respecto a los ladrillos fabricados como patrón, así como una inertización exitosa de los contaminantes adheridos a los residuos. En la investigación conducida por ElicheQuesada (2012a), en la que se reemplazó parcialmente la mezcla cruda cerámica por residuos de mármol en diferentes porcentajes en peso $(5 \%, 10 \%, 15 \%$ y $20 \%$ ), se observaron modificaciones en las propiedades físicas y mecánicas de los ladrillos en función del porcentaje de adición y de la temperatura de cocción, no encontrando apenas variaciones respecto del ladriIlo patrón con el $15 \%$ de adición.

Por otra parte, las adiciones de residuos o subproductos industriales de naturaleza orgánica y biomasas residuales producen fundamentalmente una estructura porosa mayor que en el material cerámico que los hace más ligeros y les confiere un mayor aislamiento térmico (Chiang, Chou, Hua, Chien y Cheeseman, 2009), además de aprovechar el poder calorífico de dichos residuos durante la cocción, contribuyendo a un significativo ahorro de materias primas y consumo de energía, y a la consiguiente disminución de emisiones a la atmósfera (Cusidó, Cremades y González, 2003; Kazmi et al., 2016). En consecuencia, la densidad de los productos obtenidos y sus resistencias mecánicas se ven considerablemente disminuidas, denominándose a dichos aditivos como "agentes formadores de poros».

\section{El objetivo de este trabajo ha sido la} utilización de biomasas residuales no empleadas hasta el momento como agente formador de poros en la elaboración de ecoladrillos en varios porcentajes [...], así como en diferentes tamaños, con el objeto de conseguir unas características físicas y mecánicas modificadas más acordes con los requerimientos técnicos y medioambientales actuales
Este tipo de efecto se ha constatado con los resultados aportados al conocimiento científico por diversos autores que han empleado con diferentes tipos de aditivos generadores de poros en cantidades variables de los mismos. Así, se han obtenido resultados variables, empleando productos tan diferentes como residuos de té (Demir, 2006), café, bagazo y lodos de la industria cervecera (Eliche-Quesada, Martínez-García, Martínez-Cartas, Cotes-Palomino y Corpas-Iglesias, 2011); serrín (Barbieri, Andreola, Lancellotti y Taurino, 2013; Demir, 2008; Eliche-Quesada et al., 2012a); tabaco y césped (Demir, 2008); lodos de tratamiento de aguas residuales (Chiang et al., 2009; Devant et al., 2010; Martínez-García, Eliche-Quesada, Pérez-Villarejo, Iglesias-Godino y Corpas-Iglesias, 2012); cáscara de arroz (Chiang et al., 2009; Chiang, Liao y Lu, 2012; Sutas, Mana y Pitak, 2012); semillas de uva y cerezas (Barbieri et al., 2013); leña de vid (Velasco, Ortiz, Giró, Melia y Rehbein, 2015); compost (Eliche-Quesada et al., 2012a); aguas residuales del lavado de la industria del aceite (Eliche-Quesada, Iglesias-Godino, Pérez-Villarejo y Corpas-Iglesias, 2014); orujo (La Rubia-García, Yebra-Rodríguez, Eliche-Quesada, Corpas-Iglesias y López-Galindo, 2012; Eliche-Quesada, Azevedo-Da Cunha y Corpas-Iglesias, 2015; Sutcu, Ozturk, Yalamac y Gencel, 2016); residuos forestales (Devant et al., 2010); y harina de hueso de aceituna y paja de trigo (Aouba. Bories, Coutand, Perrin y Lemercier, 2016). Incluso se han llegado a incorporar las cenizas de algunos materiales residuales de naturaleza orgánica para conseguir similares resultados, fundamentalmente cenizas de la combustión de orujo (Eliche-Quesada y Leite-Costa, 2016), de incineración de biomasa (PérezVillarejo, Eliche-Quesada, Iglesias-Godino, MartínezGarcía y Corpas-Iglesias, 2012), de caña de azúcar (Barbieri et al., 2013) o de cáscara de arroz (Kazmi et al., 2016; Sutas et al., 2012; Rahman, 1987).

El objetivo de este trabajo ha sido la utilización de biomasas residuales no empleadas hasta el momento como agente formador de poros en la elaboración de ecoladrillos en varios porcentajes, establecidos en volumen, así como en diferentes tamaños, con el objeto de conseguir unas características físicas y mecánicas modificadas más acordes con los requerimientos técnicos y medioambientales actuales y que contribuyan al cumplimiento de la Directiva 98/2008 de residuos, de la Ley 22/2011 de residuos y suelos contaminados y de la estrategia comunitaria para la economía circular que se plasmará próximamente en directiva europea. 


\section{MATERIALES Y MÉTODOS}

\subsection{Materiales}

En el estudio del comportamiento de los ecoladrillos fabricados con la inclusión de diferentes biomasas residuales se han empleado la arcilla y las biomasas residuales como agentes formadores de poros que se describen a continuación.

\subsubsection{Arcilla}

La arcilla empleada en el estudio experimental procedente de una cantera ubicada en la provincia de Granada (España) explotada por la empresa Ladrillos Suspiro del Moro, SL. La pasta arcillosa cruda contiene una mezcla de dos tipos de arcillas: arcilla grea al $40 \%$ y arcilla lima como desengrasante al $60 \%$. La tabla 1 resume las características técnicas que presenta la arcilla empleada.

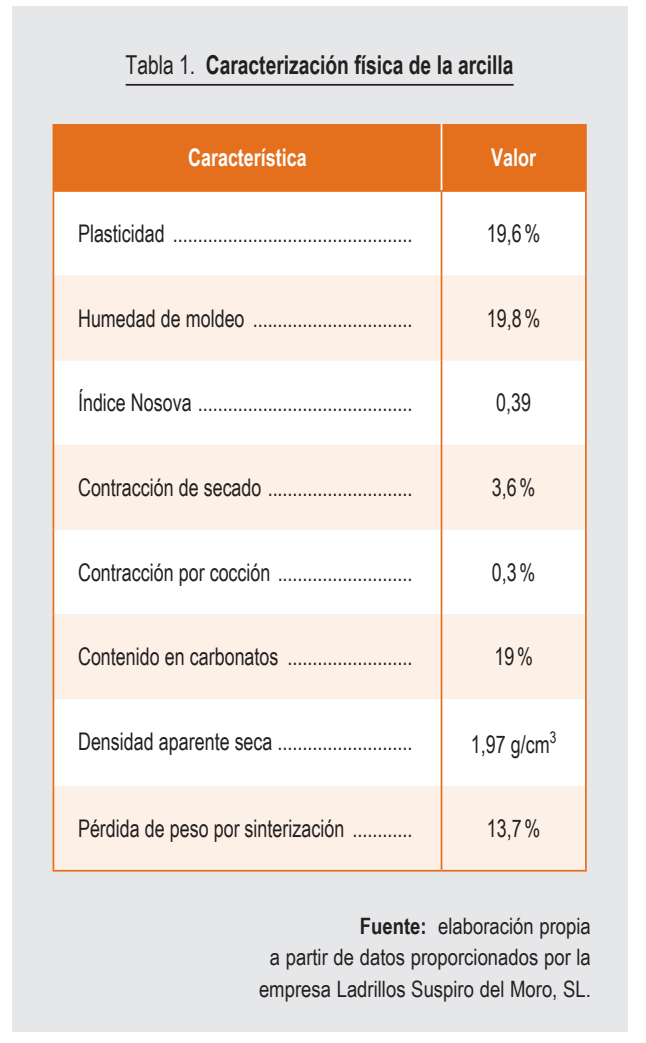

La plasticidad que presenta la mezcla de arcillas es la necesaria para conseguir pastas moldeables en crudo que demanden bajas humedades de moldeo, normalmente comprendidas entre el 18 y el $25 \%$, para que proporcionen un producto final acabado con la menor contracción posible y la adecuada resistencia mecánica. Para conseguir esa plasticidad y que la eliminación del agua libre contenida entre las partículas de la mezcla arcillosa cruda presente unas contracciones por secado adecuadas se necesita una humedad de moldeo del $19,8 \%$.

El índice de Nosova refleja la sensibilidad de una arcilla al proceso de secado mediante la relación volumétrica entre el agua perdida por la pieza y el volumen inicial de poros existentes en la misma. Índices inferiores a 0,5 son bajos y característicos de arcillas de alta porosidad en seco, con una red capilar que favorece la salida de humedad durante el proceso de secado. Índices por encima de 1 indican redes capilares muy angostas que dificultan considerablemente el proceso de secado.

La contracción por secado medida como la pérdida de agua superficial eliminada que produce el acercamiento de las partículas de la pasta cerámica hasta que esta alcanza el estado de equilibrio es necesario que se produzca antes de la cocción de la pieza al objeto de evitar tensiones y roturas en la misma. En arcillas para ladrillos y tejas, este parámetro suele variar entre el 4 y el $7 \%$, por lo que la mezcla arcillosa empleada tiene un bajo riesgo de roturas por tensiones originadas en la cocción.

Por su parte, la contracción lineal por cocción o sinterización se produce por las reacciones y los procesos físicos que pueden desarrollarse en los minerales presentes en la arcilla manifestados durante la fase de cocción que se observan en la figura 1 al realizar el análisis termodilatométrico que se describe a continuación:

- Entre la temperatura ambiente y los $500{ }^{\circ} \mathrm{C}$, se produce una dilatación del 0,53\%, asociada a la eliminación del agua ligada químicamente con la arcilla por la llamada deshidroxilación del mineral arcilloso, que no es significativa. Dilataciones hasta dicha temperatura comprendidas entre el 0,45 y el $0,55 \%$ se consideran normales en los procesos de fabricación de productos cerámicos por extrusión para que el precalentamiento de las piezas hasta esa temperatura se lleve a cabo con un riesgo bajo de fisuración. 


\section{Figura 1. Análisis termodilatométrico de la arcilla}

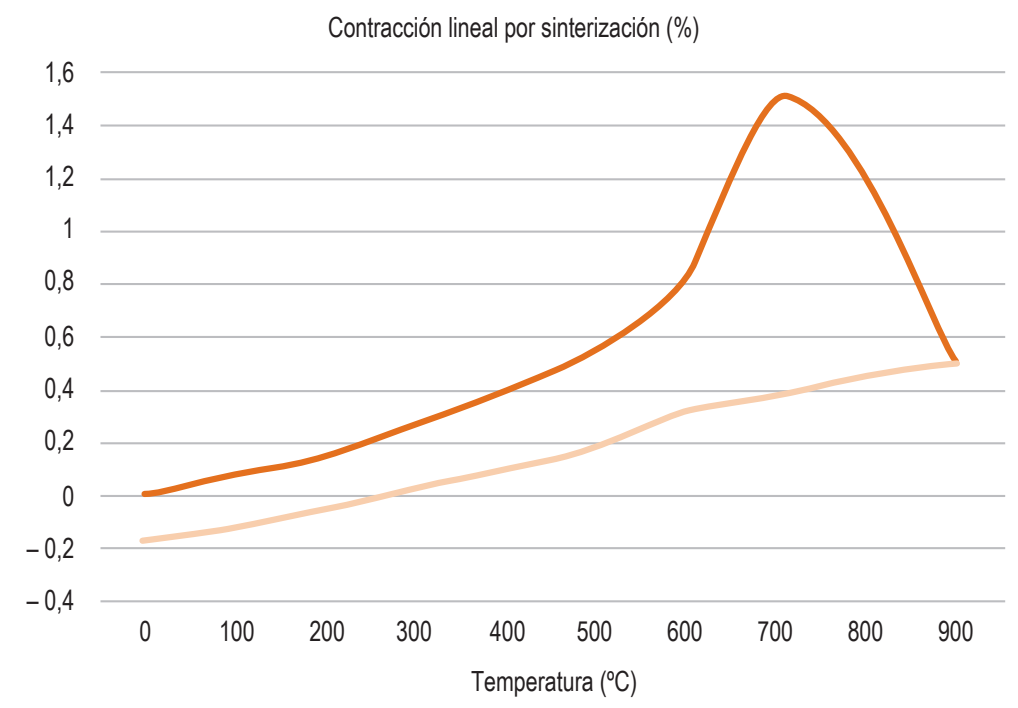

Fuente: elaboración propia a partir de datos proporcionados por la empresa Ladrillos Suspiro del Moro, SL.

- Entre 500 y $600^{\circ} \mathrm{C}$ la arcilla presenta una dilatación del $0,27 \%$ por la presencia de cuarzo libre, cuyo cambio de fase a una temperatura aproximada de $573^{\circ} \mathrm{C}$, (cuarzo $\alpha$ a cuarzo $\beta$ ) origina una dilatación que, de no producirse al mismo tiempo en toda la pieza, puede dar lugar a tensiones que lleven asociadas la aparición de fisuras.

A partir de los $600^{\circ} \mathrm{C}$, la dilatación va perdiendo intensidad, hasta llegar a estabilizarse sobre los 780$800^{\circ} \mathrm{C}$. La dilatación total sufrida durante el calentamiento de la arcilla es del $1,22 \%\left(817^{\circ} \mathrm{C}\right)$. En arcillas para ladrillos y tejas, la dilatación máxima adopta normalmente valores comprendidos entre $0,85-1,15 \%$, por lo que los valores obtenidos para la dilatación máxima de las mezclas analizadas se consideran altos. Por tanto, el precalentamiento de las mezclas formuladas deberá ser adecuado para prevenir la aparición de fisuras de precalentamiento en las piezas.

Entre los $810^{\circ} \mathrm{C}$ y los $915^{\circ} \mathrm{C}$, la curva dilatométrica presenta una contracción brusca, relacionada con la formación de fase líquida y la sinterización, adoptando un valor de $0,76 \%$.
Del ensayo termodilatométrico se observa también que la temperatura óptima de cocción para la arcilla está comprendida en el intervalo entre $850-870^{\circ} \mathrm{C}$.

El enfriamiento desde la temperatura de cocción hasta los $600{ }^{\circ} \mathrm{C}$ puede ser relativamente rápido, ya que la contracción es baja y el material se encuentra en estado piroplástico, pudiendo adaptarse sin problema a las tensiones derivadas del enfriamiento. El enfriamiento debe controlarse fundamentalmente entre 600 y $500^{\circ} \mathrm{C}$, coincidiendo con la transformación de cuarzo $\beta$ a cuarzo a para evitar defectos como el carpío o rotura por enfriamiento de las piezas cerámicas. El valor de contracción se sitúa en el 0,23\%, encontrándose en el rango de las contracciones normales $(0,20-0,25 \%)$. Desde los $500{ }^{\circ} \mathrm{C}$ hasta la temperatura ambiente, la contracción de enfriamiento se produce a menor velocidad, adoptando un valor de 0,55\%, típico de las arcillas calcáreas, cuyo valor se encuentra entre el 0,51 y el $0,60 \%$, lo cual indica que el contenido en carbonatos es significativo.

La existencia del $19 \%$ en carbonatos en la pasta es determinante para las reacciones que tienen lugar a 
altas temperaturas, generando fases cristalinas que no se producen en ausencia de ellos y que afectan en gran medida a las propiedades de la pieza cocida. La calcimetría o determinación de carbonatos es un ensayo esencial y constituye un indicativo de la porosidad de la pieza cocida, así como del incremento en el consumo térmico del horno, producido por la descomposición del $\mathrm{CaCO}_{3}$.

La densidad aparente en seco es una medida de la compacidad, que es una de las propiedades más importantes de la pieza moldeada, ya que determina su comportamiento en las diferentes etapas del proceso cerámico y ejerce una marcada influencia, tanto sobre las propiedades de la pieza cruda (resistencia mecánica, permeabilidad, etc.) como sobre las propiedades del producto cocido (tamaño, absorción de agua, resistencia mecánica, etc.). La densidad aparente que presenta la arcilla utilizada de $1,97 \mathrm{~g} / \mathrm{cm}^{3}$ pone de manifiesto una adecuada distribución entre partículas finas y gruesas que repercutirá positivamente en la resistencia mecánica de las piezas que se fabriquen con ella.

\subsubsection{Biomasas residuales}

Las biomasas residuales utilizadas como agente formador de poros en los ecoladrillos fabricados fueron proporcionadas por productores locales y regionales, y procesadas mediante cribado para obtener las tres fracciones siguientes: 0/2 mm, 0/1 mm y 1/2 mm. Resulta necesario destacar que estas biomasas se proporcionaron con un tamaño máximo de $2 \mathrm{~mm}$ que las hace inviables para su valorización energética, pues no entran en combustión en las calderas, sino que son expulsadas en forma de partículas en suspensión. La tabla 2 resume la caracterización de las 6 biomasas empleadas que se han denominado con las siguientes abreviaturas:

- Cascarilla de arroz (C).

- Cáscara de almendra (CA).

- Hueso de aceituna (HA).

- Hoja de olivo (HO).

- Leña de olivo (LO).

- Poda de olivo (PO).

Tabla 2. Caracterización de las biomasas empleadas

\begin{tabular}{|c|c|c|c|c|c|c|}
\hline & C & CA & HA & HO & LO & PO \\
\hline Humedad $(\%)$ & 8,34 & 10,38 & 7,47 & 11,02 & 7,42 & 10,89 \\
\hline Contenido en cenizas (\%) & 13,71 & 1,06 & 0,89 & 14,17 & 1,43 & 5,50 \\
\hline 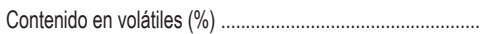 & 86,79 & 99,10 & 99,40 & 63,74 & 90,15 & 79,80 \\
\hline
\end{tabular}

Análisis elemental

\begin{tabular}{|c|c|c|c|c|c|c|}
\hline Carbono $(\%)$ & 39,8 & 49,23 & 48,02 & 45,71 & 46,49 & 47,12 \\
\hline 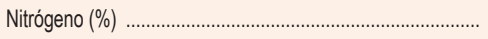 & - & 0,29 & 0,13 & 1,56 & 0,32 & 1,11 \\
\hline 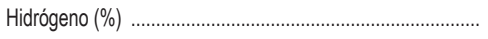 & - & 6,33 & 6,39 & 6,66 & 6,77 & 6,82 \\
\hline 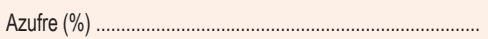 & - & $N D^{*}$ & 0,02 & 0,11 & 0,00 & 0,05 \\
\hline 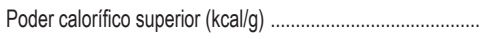 & 4.160 & 4.383 & $5.091 / 4.555$ & 4.694 & 4.189 & 4.349 \\
\hline 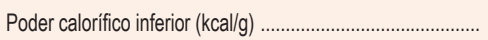 & - & 3.981 & 4.222 & 4.305 & 3.838 & 3.956 \\
\hline Contenido en cloro (\%) & 0,018 & 0,004 & 0,026 & 0,03 & 0,01 & 0,02 \\
\hline
\end{tabular}


Tabla 2. Caracterización de las biomasas empleadas (cont.)

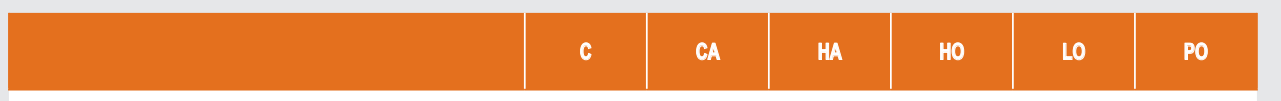

$\ldots / \ldots$

Composición de las cenizas por FRX

\begin{tabular}{|c|c|c|c|c|c|c|}
\hline $\mathrm{SiO}_{2}(\%)$ & 92,18 & 6,59 & $>$ LLD & 15,14 & 0,81 & 1,72 \\
\hline $\mathrm{Al}_{2} \mathrm{O}_{3}(\%)$ & $>$ LLD & 2,27 & $>$ LLD & 5,37 & 0,27 & 0,56 \\
\hline $\mathrm{Fe}_{2} \mathrm{O}_{3}(\%)$ & 0,15 & 0,98 & 0,07 & 3,53 & 0,77 & 0,69 \\
\hline $\mathrm{MnO}(\%)$ & 0,06 & 0,03 & $>$ LLD & 0,07 & 0,03 & 0,08 \\
\hline $\mathrm{MgO}(\%)$ & 0,63 & 3,59 & $>$ LLD & 3,69 & 2,14 & 3,85 \\
\hline $\mathrm{CaO}(\%)$ & 1,18 & 17,82 & 0,36 & 38,57 & 52,15 & 59,23 \\
\hline $\mathrm{Na}_{2} \mathrm{O}(\%)$ & 0,16 & 0,57 & 0,05 & 0,21 & 0,09 & 0,14 \\
\hline $\mathrm{K}_{2} \mathrm{O}(\%)$ & 2,45 & 37,78 & 0,50 & 5,12 & 18,44 & 8,54 \\
\hline $\mathrm{TiO}_{2}(\%)$ & 0,01 & 0,12 & 0,01 & 0,45 & 0,02 & 0,06 \\
\hline $\mathrm{P}_{2} \mathrm{O}_{5}(\%)$ & 0,50 & 3,36 & 0,05 & 2,60 & 3,00 & 3,46 \\
\hline
\end{tabular}

${ }^{*} \mathrm{~N} / \mathrm{D}$ (no disponible)

Fuente: elaboración propia a partir de los ensayos realizados.

\subsection{Métodos}

\subsubsection{Fabricación de los ladrillos}

Para la elaboración de los ecoladrillos con las diferentes biomasas como agentes formadores de poros se prepararon 10 muestras de ladrillos con la arcilla proporcionada por el fabricante, con la incorporación del $7,5,15$ y $25 \%$ en volumen de cada una de las biomasas residuales, en los tamaños $0 / 2 \mathrm{~mm}, 0 / 1 \mathrm{~mm}$ y $1 / 2 \mathrm{~mm}$, así como 10 muestras de ladrillos elaborados solamente con la arcilla, que servirán de patrón o referencia para la comparación de los resultados obtenidos en los ensayos. Es de destacar que en todas las investigaciones consultadas en la bibliografía los reemplazos de arcilla se realizaron en peso de agente formador de poros y no en volumen, por lo que la comparación entre resultados con los diferentes especímenes no resulta del todo correcta.

Los 19 tipos de mezclas preparadas se homogeneizaron en una amasadora con la adición del $5 \%$ de agua de amasado al objeto de obtener la adecuada plasticidad y cohesión durante la conformación y, posterior- mente, se moldearon en piezas de $10 \times 30 \times 60 \mathrm{~mm}$ en una prensa uniaxial de laboratorio con la presión de moldeo de 2,5 MPa, que es la empleada en la extrusión a escala industrial. Las muestras conformadas se secaron en estufa a $100^{\circ} \mathrm{C}$ durante 24 horas con el objeto de reducir el contenido de humedad y, posteriormente, se cocieron en un horno eléctrico de laboratorio durante 12 horas, de acuerdo al ciclo establecido por el fabricante, a razón de $1,22{ }^{\circ} \mathrm{C} / \mathrm{min}$ de aumento de temperatura, hasta alcanzar la temperatura máxima de cocción de $850^{\circ} \mathrm{C}$, que se mantuvo durante media hora. La figura 2 presenta la serie de probetas elaboradas con hueso de aceituna $(\mathrm{HA})$ y la muestra patrón $(\mathrm{A})$, apreciándose la diferencia de porosidad superficial con el incremento de la cantidad y el tamaño de biomasa incorporada.

Las piezas se designaron como L-x-y, donde:

- L. Es C, CA, HA, HO, LO y PO, según la inclusión de biomasa residual, o A sí se denomina al ladrillo patrón.

- x. Es 0/2, 0/1 o 1/2, según el tamaño de partícula de la biomasa residual en milímetros.

- y. Es 7,5, 15 ० 25, según el porcentaje de sustitución de arcilla por biomasa residual en volumen. 


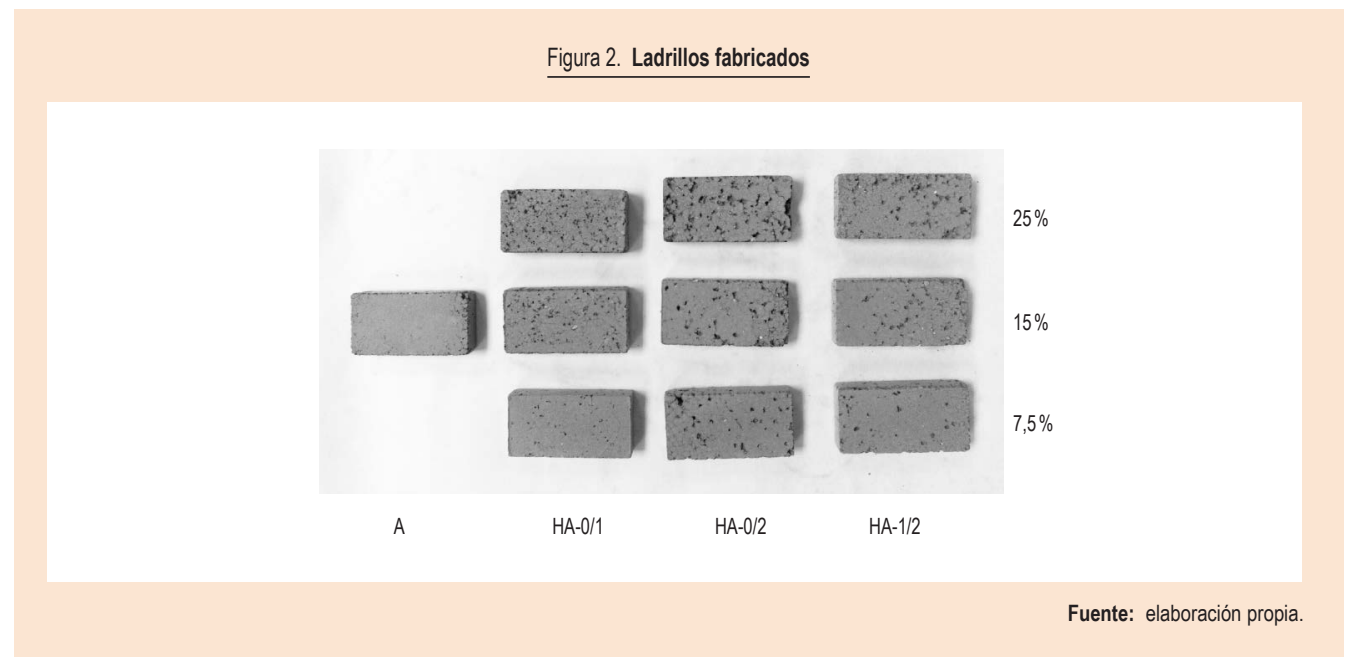

\subsubsection{Caracterización de los ladrillos}

Los ladrillos de arcilla cocida y los ecoladrillos fabricados con la incorporación de las biomasas residuales han sido sometidos a una serie de ensayos con la finalidad de caracterizarlos y conocer el efecto de la biomasa sobre los mismos. La tabla 3 recoge los ensayos realizados, así como la normativa técnica empleada para la realización del ensayo, en su caso.

Tabla 3. Ensayos realizados a los ladrillos y normativa técnica empleada

\begin{tabular}{|c|c|}
\hline \multicolumn{1}{|c|}{ Ensayo } & Normativa \\
\hline Densidad aparente ....................... & UNE-EN 772-13 \\
Absorción por capilaridad .................. & UNE-EN 772-11 \\
Absorción por inmersión total ............. & UNE-EN 772-21 \\
Volumen de poros abiertos ................. & UNE-EN 772-4 \\
Porosidad aparente .......................... & UNE-EN 772-4 \\
Pérdida de peso por sinterización ........ & - \\
\hline Resistencia a compresión .................. & UNE-EN 772-1 \\
Conductividad térmica ......................... & - \\
\hline
\end{tabular}

Fuente: elaboración propia.

\section{RESULTADOS Y DISCUSIÓN}

En el estudio del comportamiento de los ecoladrillos fabricados con las diferentes biomasas residuales se han llevado a cabo los ensayos físicos relativos a la determinación de la densidad aparente del ladrillo y las pérdidas de peso experimentadas tras la sinterización de los mismos, sus propiedades hidrofísicas, incluyendo absorción de agua por capilaridad y por inmersión total, diferentes comprobaciones asociadas a la porosidad, medidas a través de la porosidad aparente de las piezas cerámicas y el volumen de poros abiertos. Asimismo se ha determinado el comportamiento mecánico a través de su resistencia a compresión y, finalmente, su contribución al aislamiento térmico medido a través de la conductividad térmica.

\section{BIOMASAS}

RESIDUALES 
Las figuras 3 a 10 recogen los resultados obtenidos de cada uno de los parámetros estudiados, expresándose cada valor como la media aritmética de 10 probetas testadas por ensayo. Estos parámetros son analizados a continuación.

\subsection{Densidad aparente}

La densidad aparente es una medida de la compacidad, una de las propiedades más importantes de la pieza moldeada, ya que determina su comportamiento en las diferentes etapas del proceso cerámico y ejerce una marcada influencia sobre las propiedades del producto cocido.

La figura 3 muestra el valor de densidad aparente en seco obtenido para los ecoladrillos fabricados con la incorporación de las diferentes biomasas residuales, así como la de los ladrillos patrón, que servirán a modo de referencia. Los resultados ponen de manifiesto que los agentes formadores de poros en la mezcla arcillosa, que se queman y rompen durante el proceso de fabricación del material, producen un descenso en la densidad de las piezas proporcional al incremento de la biomasa (Bories, Borredon, Vedrenne y Vilarem, 2014; Bories, Aouba, Vedrenne y Vilarem, 2015; Demir, 2008). La variación que experimentan se encuentra en un rango entre $+1,72 \mathrm{y}-22,12 \%(1,623$ $\left.\mathrm{g} / \mathrm{cm}^{3}\right)$, siendo la cáscara de almendra (CA) la que proporciona las menores densidades y la cascarilla de arroz (C) las mayores. Todos los productos biomásicos procedentes del olivo han generado densidades muy similares en los ecoladrillos. Sin embargo, dentro de cada tipo de ecoladrillo no se han observado diferencias significativas en cuanto a la afección del tamaño de partícula. Resultados bastante similares se han constatado en los experimentos realizados por otros

\section{La menor densidad de los}

ecoladrillos elaborados

proporciona una notable mejora

en el manejo, en el transporte y en

la puesta en obras de los ladrillos,

así como en cuanto al peso que le

pueden conferir a la estructura de

los edificios

\section{Figura 3. Densidad aparente $\left(\mathrm{g} / \mathrm{cm}^{3}\right)$ de los ladrillos de arcilla cocida}

$\begin{array}{llllllllll}0 & 0,2 & 0,4 & 0,6 & 0,8 & 1 & 1,2 & 1,4 & 1,6 & 1,8\end{array}$

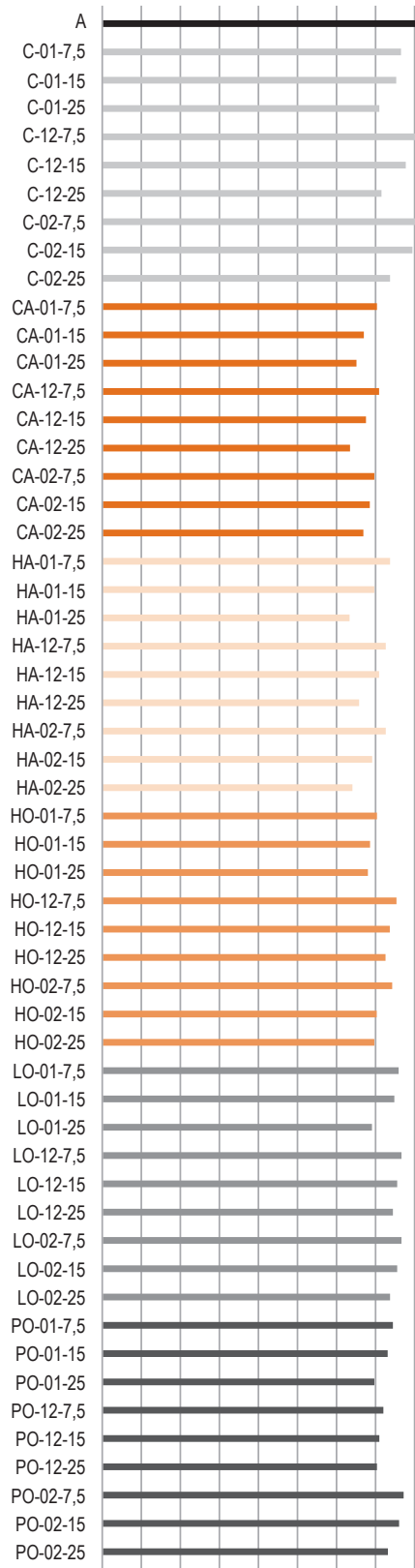

Fuente: elaboración propia. 
autores que han incorporado tanto partículas de biomasas o subproductos agrícolas orgánicos (Aouba et al., 2016; Bories et al., 2014; Bories et al., 2015; Sutcu et al., 2016) como inorgánicos (Eliche-Quesada et al., 2012a; Eliche-Quesada y Leite-Costa, 2016).

\subsection{Absorción por capilaridad}

La figura 4 muestra el valor de absorción de agua por succión capilar de los ladrillos ensayados. En general se observa un incremento proporcional de este parámetro a medida que aumenta el contenido en biomasa residual en las piezas, que se muestra en el rango entre $-1,34$ y $+61,20 \%$ con respecto a los $0,299 \mathrm{~g} / \mathrm{cm}^{2}$. min que presentan los ladrillos de referencia. Estos valores han resultado similares a los obtenidos en ladrillos elaborados con compost (Eliche-Quesada et al., 2012a) o superiores en los elaborados con serrín (Eliche-Quesada et al., 2012a); y comparados con agentes formadores de poros inorgánicos, como en las cenizas de paja de arroz, han resultado inferiores (Kazmi et al., 2016); con el polvo de mármol, superiores (Eliche-Quesada et al., 2012a); o con cenizas de orujo, prácticamente similares (ElicheQuesada y Leite-Costa, 2016). Las mayores absorciones se atribuyen a la transformación de los agentes formadores de poros orgánicos durante el proceso térmico que determinan una mayor porosidad abierta e intercomunicada en el producto cerámico final (Montero, Jordán, Almendro-Candel, Sanfeliú y Hernández-Crespo, 2009; Weng, Lin y Chiang, 2003). No obstante, a pesar de la amplitud del rango indicado, se observa una diferencia de cantidad de agua absorbida entre las muestras estudiadas en valor absoluto insignificante, por lo que se puede afirmar que no existe influencia del tipo y tamaño de partícula de la biomasa en los resultados obtenidos.

La capilaridad es un requerimiento fundamental en estos materiales de construcción, y a la vista de los resultados, y por su sistema de aplicación, se recomienda la

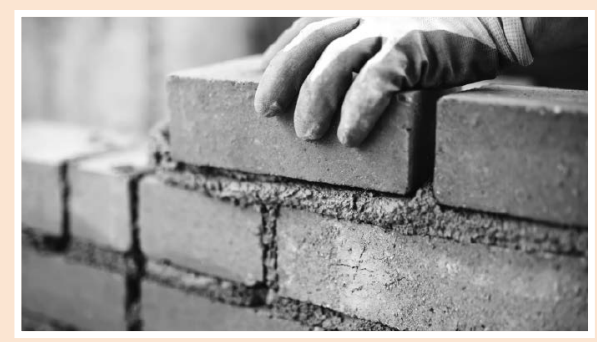

Figura 4. Absorción de agua por capilaridad $\left(\mathrm{g} / \mathrm{cm}^{2} . \mathrm{min}\right)$ de los ladrillos de arcilla cocida

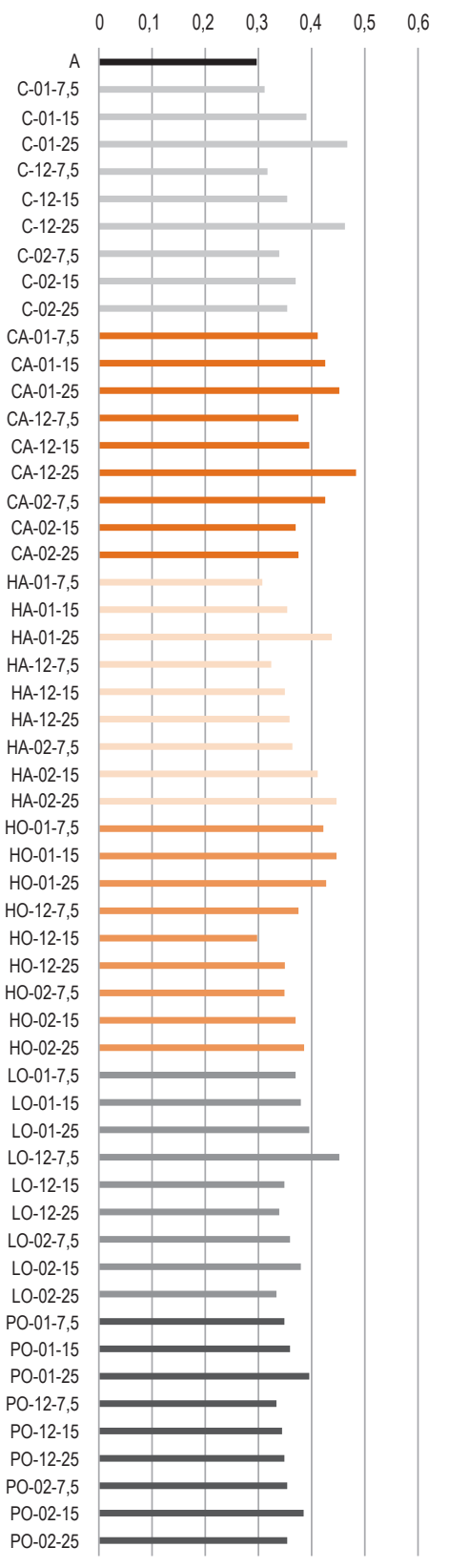

Fuente: elaboración propia. 
humectación de las piezas previa a su puesta en obra con el objeto de no modificar la consistencia del mortero, bien por succión del agua de amasado o bien por el aumento de la relación agua/cemento del mismo.

\subsection{Absorción total}

Como era de esperar, la absorción total que han experimentado los ladrillos cerámicos estudiados es alta, debido a que por naturaleza la cerámica es un material poroso, manteniendo una tendencia ascendente conforme aumenta el reemplazo de agente formador de poros (véase figura 5). Los valores oscilan entre $-3,67$ y $+37,05 \%$ respecto del ladrillo de referencia $(25,96 \%)$, encontrándose las mayores y más dispersas absorciones en los ladrillos elaborados con cascarilla de arroz (C), con cáscara de almendra (CA) y con hueso de aceituna (HA). Por otra parte, se observa que las menores y más constantes absorciones totales las proporcionan los especímenes en los que se han incorporado los subproductos del olivo como agente formador de poros ( $\mathrm{HO}, \mathrm{LO}$ y $\mathrm{PO}$ ). Estos valores están en consonancia con los obtenidos en las investigaciones conducidas por Demir (2008), Bachir y Halima (2012) y Barbieri y sus colaboradores (2013), que utilizaron tamaños de partícula de biomasa de $600 \mu \mathrm{m}$ a 1,6 mm y han resultado superiores a los obtenidos por Aouba y sus colaboradores (2016) en su trabajo de investigación con harina de hueso de aceituna.

Los valores de absorción por inmersión total obtenidos en los ecoladrillos estudiados, incluido el ladrillo patrón, son mayores del $20 \%$ máximo recomendado por algunos autores para la consecución de muros de ladrillo durables (Camino, León, Llorente y Olivar, 2014; Phonphuak y Chindaprasirt, 2015), aunque pueden ser perfectamente empleados en la ejecución de tabiques de partición interiores o en el exterior, en fachadas para revestir (Velasco et al., 2015).

Finalmente, no se aprecia que el tamaño de la partícula interfiera en el valor de la absorción total que presentan los ecoladrillos.

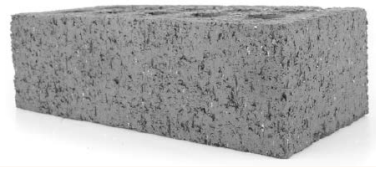

\section{Figura 5. Absorción total (\%) de agua de los ladrillos de arcilla cocida}

$\begin{array}{lllllllll}0 & 5 & 10 & 15 & 20 & 25 & 30 & 35 & 40\end{array}$

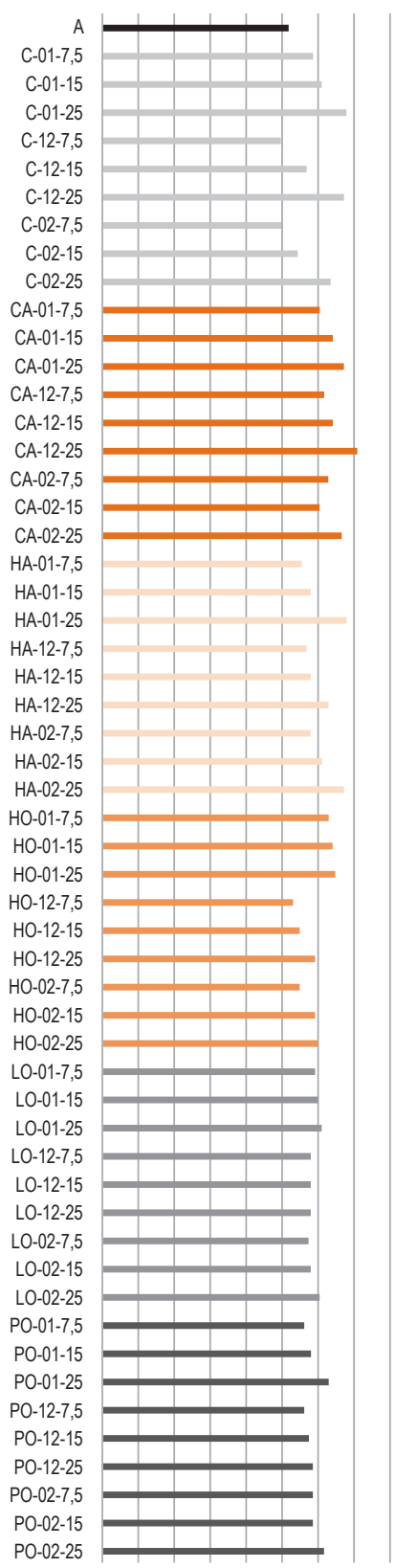

Fuente: elaboración propia. 


\subsection{Volumen de poros abiertos}

Los resultados obtenidos de porosidad abierta de los ladrillos cerámicos medida a través del volumen que ocupan sus poros abiertos a la superficie e interconectados se representan en la figura 6 , donde se observa una tendencia creciente con respecto al aumento de la cantidad de biomasa residual, con valores entre un 0,26 y un $34,47 \%$ mayores que el que presenta el ladrillo de referencia $(7,49 \%)$. Sin embargo, no se aprecian diferencias en el comportamiento en cuanto al tamaño de partícula. Esta tendencia es apenas apreciable en los ecoladrillos elaborados con los residuos de olivar (HO, LO y PO), en los que no existen diferencias significativas en cuanto al tipo de biomasa, el tamaño de partícula y el porcentaje de reemplazo. Estos resultados están muy por debajo de los reportados por Eliche-Quesada y sus colaboradores (2014).

Finalmente, hay que indicar que la cantidad y el tipo de poros abiertos están íntimamente relacionados con la capacidad de absorción de agua capilar del material, por lo que en estos casos se recomienda la humectación previa a la puesta en obra del ladrillo descrita en el apartado anterior.

\subsection{Porosidad aparente}

La porosidad aparente representa el volumen de poros abiertos respecto del volumen aparente de la pieza. Tal y como se aprecia en la figura 7, salvo en los ecoladrillos elaborados con cascarilla de arroz (C), en los que se aprecia una ligera tendencia a crecer conforme aumenta el contenido en agente formador de poros, no se observa apenas influencia del tipo, tamaño y porcentaje de reemplazo en la porosidad aparente que presentan, en muchos de los casos inferior a la del ladrillo patrón.

En consecuencia, se observa el bajo rango en el que se presentan los valores, oscilando entre $-6,17$ hasta el $14,51 \%$ respecto del valor de referencia $(42,11 \%)$. La porosidad aparente obtenida resulta algo más elevada en este estudio que en los llevados a cabo por otros investigadores (Aouba et al., 2016; Eliche-Quesada et al., 2015) debido a que estos emplearon tamaños de partículas por debajo de $150 \mu \mathrm{m}$, muy inferior a los $2 \mathrm{~mm}$ de tamaño máximo utilizados en este estudio.

Figura 6. Volumen de poros abiertos (\%) de los ladrillos de arcilla cocida

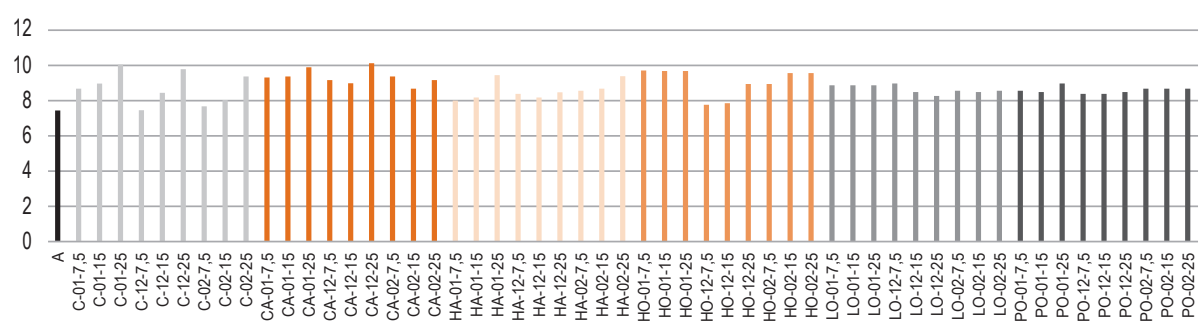

Figura 7. Porosidad aparente $(\%)$ de los ladrillos de arcilla cocida

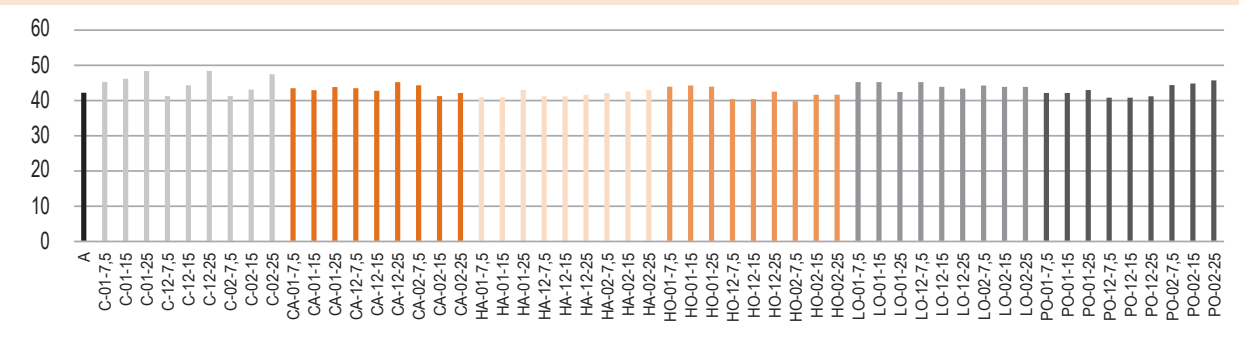

Fuente: elaboración propia. 


\subsection{Pérdida de peso tras sinterización}

La pérdida de peso por sinterización es la pérdida de masa que experimentan los ladrillos de arcilla tras su cocción y se debe a la creación de porosidad en el sistema durante el proceso de secado y cocción, fundamentalmente por la deshidroxilación y carbonatación de los componentes de las arcillas (Bories et al., 2014). En el caso de los ecoladrillos se debe además a la formación porosa por la combustión y volatilización de los componentes orgánicos de las biomasas empleadas, tales como la celulosa, la hemicelulosa y la lignina (Sutcu et al., 2016; ElicheQuesada et al., 2012a). Esta formación porosa produce la pérdida de peso, ya que el aire es más ligero que la matriz arcillosa y los agentes formadores de poros.

Los resultados obtenidos que se muestran en la figura 8 ponen de manifiesto que este parámetro crece proporcionalmente con el incremento de la sustitución de biomasa, fundamentalmente en el caso de la cáscara de almendra $(\mathrm{CA})$ y del hueso de aceituna $(\mathrm{HA})$, pero es independiente del tamaño de la misma, salvo en los residuos biomásicos de cascarilla de arroz (C), cáscara de almendra (CA) y, principalmente, hoja de olivo $(\mathrm{HO})$, donde para el tamaño 02 se ha visto reducida sensiblemente la pérdida de peso. Aouba y sus colaboradores (2016) también encontraron determinante el factor tamaño de partícula en las pérdidas de peso que se produjeron en sus ladrillos elaborados con harina de hueso de aceituna. En general, para esta propiedad se observan una amplitud de resultados con valores que oscilan entre - 41,00 y 44,04\% respecto del ladrillo patrón (15,28\%). En la revisión bibliográfica realizada se han encontrado pérdidas de peso tras la sinterización de los ecoladrillos similares (Aouba et al., 2016; Eliche-Quesada y Leite-Costa, 2016) y superiores (Eliche-Quesada, Martínez-Martínez, Pérez-Villarejo e Iglesias-Godino, 2012b; Bories et al., 2014), que pueden ser atribuidas a la pérdida de peso experimentada por la calcinación de las biomasas, además de al alto valor experimentado por los ladrillos patrón, que está prácticamente en el límite recomendado (15\%) para reducir el efecto negativo en la resistencia a compresión de los productos cerámicos (Weng et al., 2003).
Figura 8. Pérdida de peso tras sinterización (\%) de los ladrillos de arcilla cocida

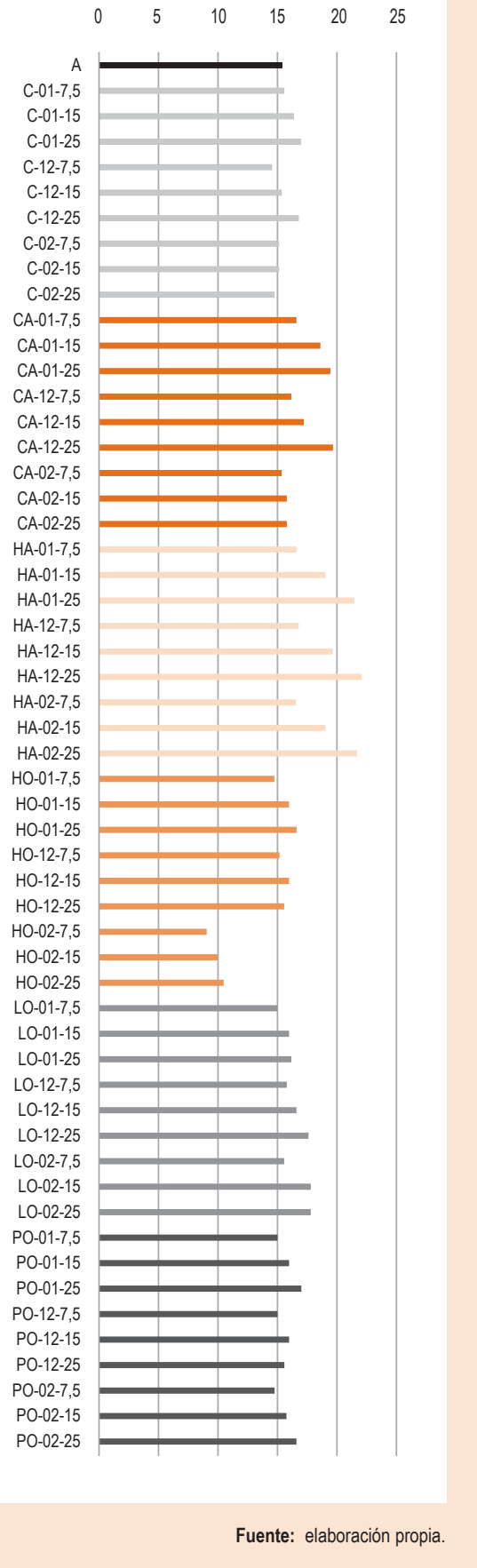




\subsection{Resistencia a compresión}

La capacidad resistente de los ladrillos, medida a través de su resistencia a compresión, es el único parámetro contemplado en la normativa técnica (CTE, 2006), siendo fundamental para conseguir la calidad necesaria en sus aplicaciones constructivas. La resistencia a compresión de todas las muestras ensayadas, medida a través de sus valores medios, se recoge en la figura 9, mostrando que los ladrillos fabricados a modo de referencia presentan una alta resistencia a compresión (62,78 MPa) comparada con las muestras elaboradas con los diferentes agentes formadores de poros. La capacidad resistente de estas se ha visto considerable y proporcionalmente disminuida a medida que se ha incorporado la biomasa residual, pero sin influencia alguna del tamaño de la misma, con valores que oscilan entre el 78,16 y el $35,82 \%$, estando sus valores relativos contemplados entre 13,71 y $40,30 \mathrm{MPa}$. Estos resultados están en consonancia con los obtenidos en los trabajos de investigación desarrollados con diferentes biomasas residuales, tales como los conducidos por Bories (2014), ElicheQuesada (2012a, 2012b, 2014) y Muñoz-Velasco (2014); y han resultado mayores que los resultados obtenidos en otras investigaciones (Demir, 2008; Kazmi et al., 2016; Phonphuak y Chindaprasirt, 2015; Velasco et al., 2015). A pesar de que los agentes formadores de poros de naturaleza orgánica proporcionan menores resistencias mecánicas que los inorgánicos, los resultados obtenidos en este estudio son similares a los alcanzados por Dondi y sus colaboradores (2009) e incluso mayores que los encontrados en el estudio comandado por Munir (2018).

Aunque se han conseguido resultados muy similares por tamaño y proporción de biomasa adicionada, son los ladrillos elaborados con cáscara de almendra (CA) y hueso de aceituna (HA) los que han visto mermadas sus resistencias en mayor medida. Resulta bastante evidente que, a pesar de no observarse diferencias significativas en cuanto al tamaño de la biomasa, el reemplazo del $25 \%$ ha afectado notablemente el comportamiento mecánico de las muestras ensayadas.

No obstante, todos los valores de resistencia mecánica a compresión están por encima de los $5 \mathrm{MPa}$ mínimos que establece el Documento Básico de Seguridad de Fachadas del Código Técnico de la Edificación (CTE, 2006).
Figura 9. Resistencia a compresión (MPa) de los ladrillos de arcilla cocida

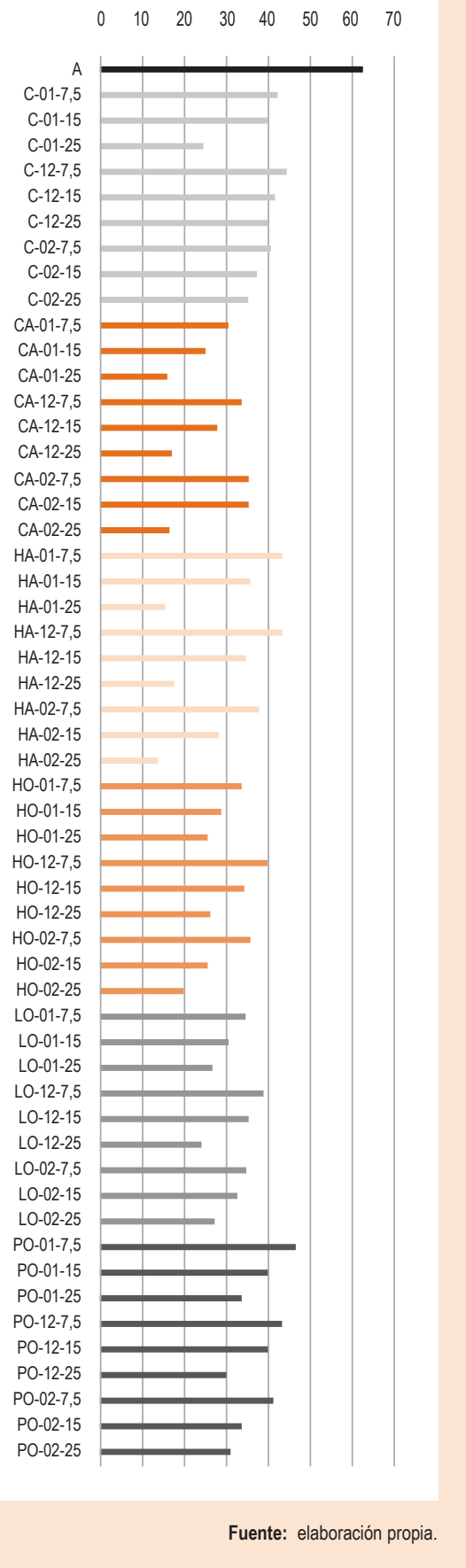




\subsection{Conductividad térmica}

La capacidad de que un sistema constructivo permita el paso de un flujo de temperatura a su través se determina mediante su transmitancia térmica según establece el Código Técnico de la Edificación en su Documento Básico DB-HE: Ahorro de Energía (CTE, 2006). Una vez analizados los parámetros cuyos resultados se han discutido en los apartados anteriores de esta sección, y en vista de que el tamaño de las partículas no es determinante para el comportamiento de los ecoladrillos, se ha procedido al estudio de la conductividad térmica de las piezas elaboradas exclusivamente con la fracción granulométrica $0 / 2$, que resultará la más favorable de uso a escala industrial por el bajo tratamiento que necesita para reducir su tamaño. La figura 10 recoge los resultados de conductividad térmica de los ecoladrillos, además de la del ladrillo patrón, observándose, en contra de lo esperado, que las mejoras en la conducción de calor se producen en las piezas elaboradas con el $7,5 \%$ de biomasa residual como agente formador de poros en volumen, que es el menor porcentaje empleado en las mezclas arcillosas. No obstante, con respecto al ladrillo patrón $(0,531$ $\mathrm{W} / \mathrm{m}$.K) prácticamente todas las muestras presentaron conductividades térmicas mejoradas, con valores entre

\section{Figura 10. Conductividad térmica (W/m.K) de los ladrillos de} arcilla cocida

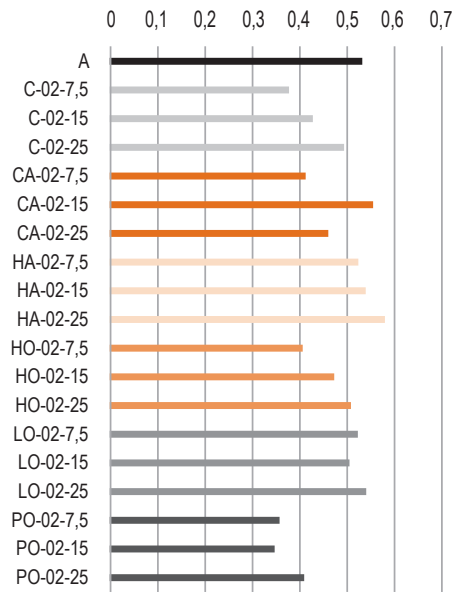

Fuente: elaboración propia

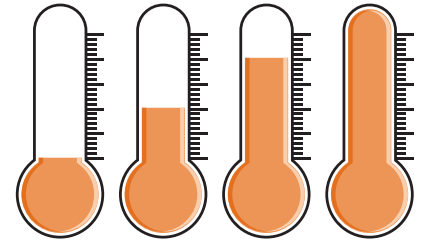

$-34,27$ y $+9,04 \%$, excepto los ecoladrillos fabricados con hueso de aceituna (HA) y cáscara de almendra (CA) en la concentración del $15 \%$ en volumen. Los estudios consultados ponen de manifiesto que estos resultados están en consonancia con los obtenidos por otros autores que han empleado tanto agentes formadores de poros orgánicos (Aouba et al., 2016; Sani y Nzihou, 2017) como inorgánicos (Munir et al., 2018).

En general, la poda de olivo (PO) es la biomasa que mejores prestaciones térmicas presenta en este estudio, seguida de la cascarilla de arroz (C), la hoja de olivo (HO) y la cáscara de almendra (CA); y el hueso de aceituna (HA) el que aporta peor comportamiento.

\section{CONCLUSIONES}

En este estudio se ha mostrado que es posible obtener ladrillos con propiedades modificadas mediante la inclusión de diferentes biomasas residuales procedentes del sector agroindustrial en la matriz arcillosa, denominados «ecoladrillos».

Basándonos en los resultados experimentales obtenidos, se pueden establecer las siguientes conclusiones:

- En general se puede concluir que, mientras que el porcentaje de agente formador de poros empleados en sustitución parcial de la mezcla arcillosa afecta significativa y proporcionalmente a todas las características de los ecoladrillos analizados, la distribución granulométrica de las partículas no ha resultado de influencia en ninguna de ellas. En consecuencia, desde el punto de vista de su aplicación a escala industrial se pueden emplear todas las biomasas residuales estudiadas sin necesidad de un tratamiento previo de reducción considerable de tamaño, lo que implicaría un ahorro de costes en el proceso. 
- En cuanto a la repercusión de las biomasas incorporadas en el comportamiento de los ecoladrillos, indicar que los fabricados con la cascarilla de arroz (C) han presentado la mayor densidad respecto a los ladrillos de referencia. Por su parte, los residuos de la industria olivarera (HA, HO, LO y PO), han producido los mejores resultados en cuanto a la absorción capilar y por inmersión total, volumen de poros abiertos, porosidad aparente, pérdida de peso por sinterización y resistencia mecánica; aunque la cascarilla de arroz $(\mathrm{C})$ también ha sido una de las biomasas residuales que ha contribuido a conseguir las mejores resistencias mecánicas. Finalmente, la poda de olivo (PO) ha ofrecido la menor conductividad térmica de todos los materiales cerámicos elaborados.

- Particularmente, la menor densidad, debido a su mayor porosidad y pérdida de peso por la sinterización de los componentes de los ecoladrillos elaborados, proporcionan una notable mejora en el manejo, en el transporte y en la puesta en obras de los ladrillos, así como en cuanto al peso que le pueden conferir a la estructura de los edificios.

- El aspecto más negativo que presentan estos materiales cerámicos es su elevada capacidad de absorción de agua, tanto capilar como por inmersión total. A pesar de ello, los valores obtenidos no comprometen su funcionalidad, ya que el ladrillo cerámico tradicional de por sí es altamente poroso y, por tanto, absorbente, y esta deficiencia se suele paliar en la práctica humectándolo antes de su puesta en obra al objeto de no modificar la consistencia del mortero fresco, tanto por succión de agua de amasado como por liberación del agua con la que se ha mojado el ladrillo.

- Los valores de resistencia a compresión de los ecoladrillos, aunque menores que los de la mezcla

\section{[...] mientras que el porcentaje de agente formador de poros empleados en sustitución parcial de la mezcla arcillosa afecta significativa y proporcionalmente a todas las características de los ecoladrillos analizados, la distribución granulométrica de las partículas no ha resultado de influencia en ninguna de ellas}

arcillosa tradicional, no comprometen la funcionalidad de los elementos constructivos que con ellos se conformen, pues superan holgadamente el valor límite establecido en la normativa técnica.

- La capacidad de aislamiento térmico de los ecoladrillos se mejora en casi un 35\% con respecto del ladrillo convencional, aunque, contrariamente a lo esperado, es la menor proporción de biomasa residual $(7,5 \%)$ la que proporciona los mejores resultados en todos los casos.

Finalmente, el empleo de biomasas residuales como agente formador de poros en las mezclas arcillosas destinadas a la producción de ladrillos cerámicos presenta como ventajas medioambientales fundamentales la valorización de un residuo que por su tamaño no tiene posibilidad de valorización energética y su única opción es el depósito en vertedero, además de la disminución en la utilización de arcilla como materia prima primaria.

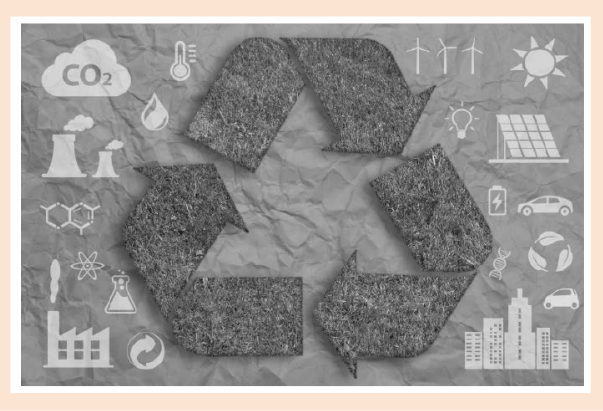

\section{El aspecto más negativo que presentan estos materiales cerámicos es su elevada capacidad de absorción de agua, tanto capilar como por inmersión total}




\section{BIBLIOGRAFÍA}

Alonso-Santurde, R., Andrés, A., Viguri, J. R., Raimondo, M., Guarini, G., Zanelli , C. y Dondi, M. (2011). Technological behaviour and recycling potential of spent foundry sandsin clay bricks. Journal of Environmental Management, 92(3), 994-1.002.

Aouba, L., Bories, C., Coutand, M., Perrin, B. y Lemercier, H. (2016). Properties of fired clay bricks with incorporated biomasses: cases of olive stone flour and wheat straw residues. Construction and Building Materials, 102(1), 7-13.

Arsenovic, M., Radojevic, Z. y Stankovic, S. (2012). Removal of toxic metals from industrial sludge by fixing in brick structure. Construction and Building Materials, 37, 7-14.

Bachir, C. y Halima, C. (2012). Effect of adding sawdust on mechanical-physiscal properties of ceramic bricks to obtain lightweight building material. World Academic Science Engineering Technology, 71, 1.608-1.612.

Banhidi, V. y Gömze, L. A. (2008). Improvement of insulation properties of conventional brick products. Materials Science Forum, 589, 1-6.

Barbieri, L., Andreola, F., Lancellotti, I. y Taurino, R. (2013). Management of agricultural biomass wastes: preliminary study on characterization and valorisation in clay matrix bricks. Waste Management, 33(11), 2.307-2.315.

Bories, C., Borredon, M. E., Vedrenne, E. y Vilarem, G. (2014). Development of eco-friendly porous fired clay bricks using pore-forming agents. A review. Journal of Environmental Management, 143, 186-196.

Bories, C., Aouba, L., Vedrenne, E. y Vilarem, G. (2015). Fired clay bricks using agricultural biomass wastes: study and characterization. Construction and Building Materials, 91, 158-163.

Brongniart, A. (1844). Traité des arts céramiques ou des poteries considérés dans leur histoire, leur pratique et leur théorie. T 1. Paris: A. Mathias.

Camino, M. S., León, F. J., Llorente, A. y Olivar, J. M. (2014). Evaluation of the behavior of brick tile masonry and mortar due to capillary rise of moisture. Materiales de Construcción, 64(314), 1-10.

Chabat, P. (1881). La brique et la terre cuite: etude historique de l'emploi de ces materiaux, fabrication et usages, motifs de construction et de decoration choisisdans l'architecture des differents peuples. Avec la collaboration de Félix Monmory. Serie 1. Paris: May et Motteroz.
Chiang, K. Y., Chou, P. H., Hua, C. R., Chien, K. L. y Cheeseman, C. (2009). Lightweight bricks manufactured from water treatment sludge and rice husks. Journal of Hazardous Materials, 171(1-3), 76-82.

Chiang, K. Y., Liao, C. K. y Lu, C. H. (2012). The effects of prepared iron-based catalyst on the energy yield in gasification of rice straw. International Journal of $\mathrm{Hy}$ drogen Energy, 41(46), 21.747-21.754.

CTE (2006). Real Decreto 314/2006, por el que se aprueba el Código Técnico de la Edificación. (BOE núm. 74, de 28 de marzo de 2006). Madrid: Ministerio de Fomento.

Cusidó, J. A., Cremades, L. V. y González, M. (2003). Cusidó gaseous emissions from ceramics manufactured with urban sewage sludge during firing processes. Waste Management, 23(3), 273-280.

Demir, I. (2006). An investigation on the production of construction brick with processed waste tea. Building and Environment, 41(9), 1.274-1.278.

Demir, I. (2008). Effect of organic residues addition on the technological properties of clay bricks. Waste Management, 28(3), 622-627.

Demir, I. y Orhan, M. (2003). Reuse of waste bricks in the production line. Building and Environment, 38, 1.451-1.455.

Demir, I., Serhat Baspinar, M. y Orhan, M. (2005). Utilization of kraft pulp production residues in clay brick production. Building and Environment, 40, 1.533-1.537.

Devant, M., Cusidó, J. A. y Soriano, C. (2010). Custom formulation of red ceramics with clay, sewage sludge and forest waste. Applied Clay Science, 53(4), 669-675.

Dondi, M., Guarini, G., Raimondo, M. y Zanelli, C. (2009). Recycling PC and TV waste glass in clay bricks and roof tiles. Waste Management, 29, 1.945-1.951.

Eliche-Quesada, D., Martínez-García, C., Martínez-Cartas, M. L., Cotes-Palomino, M. T.y Corpas-Iglesias, F.A. (2011). The use of different forms of waste in the manufacture of ceramic bricks. Applied Clay Science, 52(3), 270-276.

Eliche-Quesada, D., Corpas-Iglesias, F. A., Pérez-Villarejo, L. e Iglesias-Godino, F. J. (2012a). Recycling of sawdust, spent earth from oil filtration, compost and marble residues for brick manufacturing. Construction and Building Materials, 34, 275-284.

Eliche-Quesada, D., Martínez-Martínez, S., Pérez-Villarejo, L. e Iglesias-Godino, F. J. (2012b). Valorization 
of biodiesel production residues in making porous clay brick. Fuel Processing Technology, 103, 166-173.

Eliche-Quesada, D., Iglesias-Godino, F. J., Pérez-Villarejo, L. y Corpas-Iglesias, F. A. (2014). Replacement of the mixing fresh water by wastewater olive oil extraction in the extrusion of ceramic bricks. Construction and Building Materials, 68, 659-666.

Eliche-Quesada, D., Azevedo-Da Cunha, R. y CorpasIglesias, F. A. (2015). Effect of sludge from oil refining industry or sludge from pomace oil extraction industry addition to clay ceramics. Applied Clay Science, 114, 202-211.

Eliche-Quesada, D. y Leite-Costa, J. (2016). Use of bottom ash from olive pomace combustion in the production of eco-friendly fired clay bricks. Waste Management, 48, 323-333.

Kazmi, S. M. S, Abbas, S., Saleem, M. A., Munir, M. J. y Khitab, A. (2016). Manufacturing of sustainable clay bricks: utilization of waste sugarcane bagasse and rice husk ashes. Construction and Building Materials, 120, 29-41.

Lajo Pérez, R. (1990). Léxico de arte. Madrid, España: Akal.

Martínez-García, C., Eliche-Quesada, D., Pérez-Villarejo, L., Iglesias-Godino, F. J. y Corpas-Iglesias, F. A. (2012). Sludge valorization from wastewater treatment plant to its application on the ceramic industry. Journal of Environmental Management, 95, 349-354.

Montero, M. A., Jordán, M. M., Almendro-Candel, M. B., Sanfeliú, T. y Hernández-Crespo, M. S. (2009). The use of a calcium carbonate residue from the stone industry in manufacturing of ceramic tile bodies. Applied Clay Science, 43, 186-189.

Munir, M. J., Kazmi, S. M. S., Wu, Y. F., Hanif, A., Khan, M. U. A. (2018). Thermally efficient fired clay bricks incorporating waste marble sludge: an industrial-scale study. Journal of Cleaner Production, 183, 487-494.

Muñoz Velasco, P., Morales Ortiz, M. P., Mendívil Giró, M. A. y Muñoz Velasco, L. (2014). Fired clay bricks manufactured by adding wastes as sustainable construction material-A review. Construction and Building Materials, 63, 97-107.

NBE-FL 90 (1990). Real Decreto 1723/1990, de 20 de diciembre, por el que se aprueba la norma básica de la edificación NBE FL-90: «Muros resistentes de fábrica de ladrillo». (BOE núm. 4, de 4 de enero de 1991). Madrid: Ministerio de Obras Públicas y Urbanismo.
Pérez-Villarejo, L., Eliche-Quesada, D., Iglesias-Godino, F. J., Martínez-García, C. y Corpas-Iglesias, F. A. (2012). Recycling of ash from biomass incinerator in clay matrix to produce ceramic bricks. Journal of Environmental Management, 95(S), 349-354.

Pérez-Villarejo, L., Martínez-Martínez, S., Carrasco-Hurtado, B., Eliche-Quesada, D., Ureña-Nieto, C. y Sánchez-Soto, P. J. (2015). Valorization and inertization of galvanic sludge waste in clay bricks. Applied Clay Science, 105-106, 89-99.

Phonphuak, N. y Chindaprasirt, P. (2015). 6: Types of waste, properties, and durability of pore-forming wastebased fired masonry bricks. Eco-Efficient Masonry Bricks and Blocks, 103-127.

Rahman M. A. (1987). Properties of clay-sand-rice husk ash mixed bricks. International Journal of Cement Composites and Lightweight Concrete, 9(2), 105-108.

Rubia-García, M. D. la, Yebra-Rodríguez, A., ElicheQuesada, D., Corpas-Iglesias, F. A. y López-Galindo, A. (2012). Assessment of olive mill solid residue (pomace) as an additive in lightweight brick production. Construction and Building Materials, 36, 495-500.

Sani, R. y Nzihou, A. (2017). Production of clay ceramics using agricultural wastes: study of properties, energy savings and environmental indicators. Applied Clay Science, 146(15), 106-114.

Sengupta, P., Saikia, N. y Borthakur, P. C. (2002). Bricks from petroleum effluent treatment plant sludge: properties and environmental characteristics. Journal of Environmental Engineering, 128(11), 1.090-1.094.

Sutas, J., Mana, A. y Pitak, L. (2012). Effect of rice husk and rice husk ash to properties of bricks. Procedia Engineering, 32, 1.061-1.067.

Sutcu, M., Ozturk, S., Yalamac, E. y Gencel, O. (2016). Effect of olive mill waste addition on the properties of porous fired clay bricks using Taguchi method. Journal of Environmental Management, 181, 185-192.

Velasco P. M., Ortiz, M. P. M., Giró, M. A. M., Melia, D. M. y Rehbein, J. H. (2015). Development of sustainable fired clay bricks by adding kindling fromvine shoot: Study of thermal and mechanical properties. Applied Clay Science, 107, 156-164.

Weng, C. H., Lin, D. F. y Chiang, P. C. (2003). Utilization of sludge as brick materials. Advances in Environmental Research, 7, 679-685. 


\section{Relación de ciclos formativos de grado superior para cursar grados en la UDIMA}

La Universidad a Distancia de Madrid (UDIMA) tiene aprobado el reconocimiento de los ciclos formativos de grado superior para cursar las carreras universitarias indicadas por la Consejería de Educación de la Comunidad de Madrid:

\section{Para el Grado en Ingeniería de Organización industrial}

- Administración y Finanzas.

- Administración de Sistemas Informáticos.

- Automatización y Robótica Industrial.

- Automoción.

- Construcciones Metálicas.

- Desarrollo de Productos Electrónicos.

- Desarrollo de Proyectos Mecánicos.

- Diseño en Fabricación Mecánica.

- Instalaciones Electrotécnicas.

- Mantenimiento Aeromecánico.

- Mantenimiento de Equipo Industrial.

- Mantenimiento y Montaje de Instalaciones de Edificio y Proceso.

- Mantenimiento de Instalaciones Térmicas y de Fluidos.

- Mecatrónica Industrial.

- Industrias de Proceso Químico.

- Producción por Mecanizado.

- Programación de la Producción en Fabricación Mecánica.

- Química Industrial.

- Sistemas Electrotécnicos y Automatizados.

- Sistemas de Regulación y Control Automáticos.

- Sistemas de Telecomunicaciones e Informáticos.
Para el Grado en Ingeniería de Tecnologías y Servicios de Telecomunicación

- Administración de Sistemas Informáticos.

- Automatización y Robótica Industrial.

- Desarrollo de Aplicaciones Informáticas.

- Desarrollo de Aplicaciones Multiplataforma.

- Desarrollo de Aplicaciones Web.

- Desarrollo de Productos Electrónicos.

- Instalaciones Electrotécnicas.

- Mantenimiento Electrónico.

- Sistemas Electrotécnicos y Automatizados.

- Sistemas de Regulación y Control Automáticos.

- Sistemas de Telecomunicaciones e Informáticos

Para los Grados en Derecho o en Ciencias del Trabajo, Relaciones Laborales y Recursos Humanos

- Administración y Finanzas.

- Asistencia a la Dirección.

- Secretariado. 


\section{para cursar grados en la UDIMA}

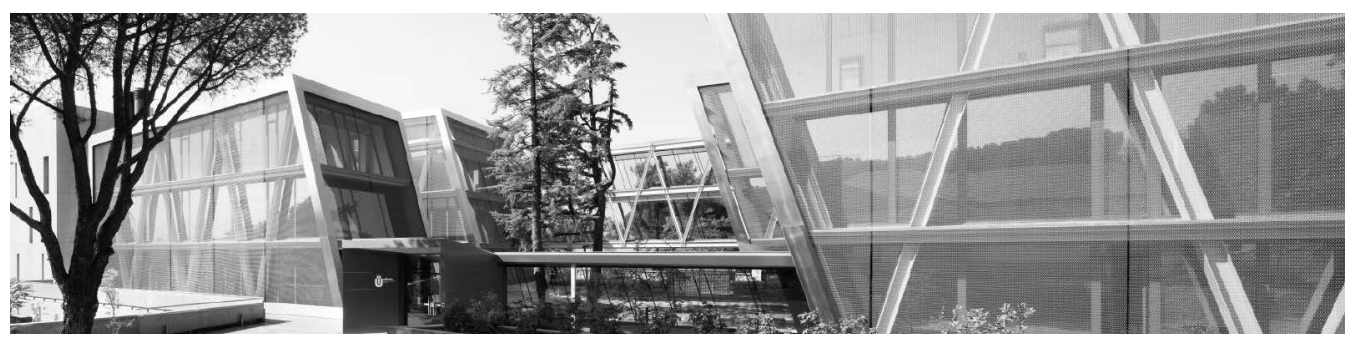

\section{< > Para el Grado en Ingeniería Informática}

- Administración de Sistemas Informáticos.

- Automatización y Robótica Industrial.

- Desarrollo de Aplicaciones Informáticas.

- Desarrollo de Aplicaciones Multiplataforma.

- Desarrollo de Aplicaciones Web.

- Sistemas de Telecomunicaciones e Informáticos.

\section{Para los Grados en Administración y Dirección de Empresas o en Economía}

- Administración y Finanzas.

- Asistencia a la Dirección.

- Comercio Internacional.

- Gestión Comercial y Marketing.

- Marketing y Publicidad.

- Secretariado.

- Transporte y Logística.

\section{Para el Grado en Marketing}

- Administración y Finanzas.

- Comercio Internacional.
- Gestión Comercial y Marketing.

- Gestión de Alojamientos Turísticos.

- Gestión de Ventas y Espacios Comerciales.

- Marketing y Publicidad.

- Transporte y Logística.

Para los Grados en Magisterio de Educación Infantil y de Educación Primaria

- Animación de Actividades Físicas y Deportivas.

- Educación Infantil.

- Integración Social.

- Animación Sociocultural y Turística.

\section{Para el Grado en Empresas y} Actividades Turísticas

- Gestión Comercial y Marketing.

- Gestión de Ventas y Espacios Comerciales.

- Gestión de Alojamientos Turísticos.

- Agencias de Viajes y Gestión de Eventos.

- Guía, Información y Asistencias Turísticas. 


\section{5 Servicio de Bolsa de Trabajo y Orientación de Prácticas Externas \\ Apoyo permanente en el desarrollo profesional a los más de 500.000 alumnos que han pasado por el CEF.- y la UDIMA}

El EF.- y la UDIMA ponen a disposición de los estudiantes diversos servicios de asesoramiento y formación para apoyar al alumno en su desarrollo académico y profesional. Es muy importante que el estudiante conozca la realidad laboral de su grado o máster, por lo que para finalizar sus estudios universitarios resulta imprescindible la realización de prácticas externas. Este servicio de Bolsa de Trabajo y Orientación de Prácticas Externas ofrece a las empresas y entidades educativas las herramientas necesarias para satisfacer sus necesidades en los procesos de selección de profesionales cualificados.

Desde nuestros orígenes hemos tenido como lema «formación para el empleo», por ello consideramos este servicio como un pilar fundamental para nuestras acciones formativas, pues todas van dirigidas a buscar empleo para nuestros alumnos, la mejora del que ya tienen o a conseguir su consolidación.
Desde el Servicio de Bolsa de Trabajo y Orientación de Prácticas Externas agradecemos tanto a las empresas y entidades educativas como a los alumnos y antiguos alumnos que utilicen este servicio como fuente de reclutamiento para los procesos de selección en todas las áreas que se imparten en el EFF.- y en la UDIMA.

Este servicio es GRATUITO tanto para los estudiantes como para las entidades educativas.

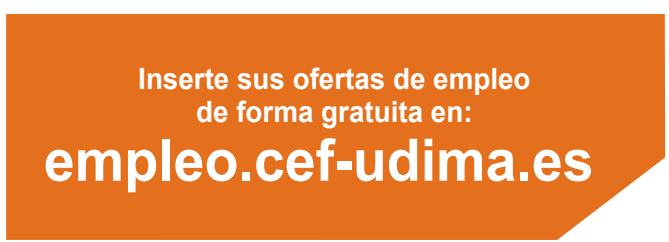

